\title{
Incidence and risk factors of pneumothorax following pre-procedural ultrasound-guided thoracentesis
}

\author{
Liran Shechtman ${ }^{1}$, Maayan Shrem $^{1}$, Yeruham Kleinbaum², Gil Bornstein ${ }^{3}$, Lee Gilad ${ }^{1}$, Chagai Grossman ${ }^{1}$ \\ ${ }^{1}$ Department of Internal Medicine F, ${ }^{2}$ Department of Diagnostic Imaging, The Chaim Sheba Medical Center, Tel-Hashomer, Ramat Gan, affiliated \\ to Sackler Faculty of Medicine, Tel-Aviv University, Tel Aviv, Israel; ${ }^{3}$ Department of Internal Medicine B, Tel Aviv Sourasky Medical Center, \\ affiliated to Sackler Faculty of Medicine, Tel-Aviv University, Tel Aviv, Israel \\ Contributions: (I) Conception and design: C Grossman; (II) Administrative support: G Bornstein; (III) Provision of study materials or patients: Y \\ Kleinbaum, L Gilad; (IV) Collection and assembly of data: L Shechtman, M Shrem; (V) Data analysis and interpretation: C Grossman, L Shechtman; \\ (VI) Manuscript writing: All authors; (VII) Final approval of manuscript: All authors. \\ Correspondence to: Chagai Grossman, MD. Department of Internal Medicine F, The Chaim Sheba Medical Center, Tel Hahsomer, 52621, Tel Aviv, \\ Israel. Email: chagaigr@gmail.com.
}

\begin{abstract}
Background: Data regarding the incidence and risk factors of pneumothorax following pre-procedural ultrasound (US)-guided thoracentesis is scarce. We aimed to evaluate the incidence and risk factors of pneumothorax following pre-procedural US-guided thoracentesis in a tertiary medical center.

Methods: Retrospective analysis of patients who underwent pre-procedural US-guided thoracentesis in Sheba Medical Center between January 2016 and December 2018. Data collected included incidence of pneumothorax following thoracentesis, baseline clinical and demographic characteristics, and thoracentesisassociated factors. Outcomes evaluated included length of hospital stay, mortality, chest tube insertion and intensive care unit admission.

Results: A total of 550 patients with pleural effusions underwent pre-procedural US-guided thoracentesis. Sixty-six (12\%) of them developed pneumothorax. Compared to patients who did not develop pneumothorax, those who developed pneumothorax had a higher rate of congestive heart failure $(32.2 \% v s .47 \%, \mathrm{P}=0.026)$, a smaller depth of pleural fluid marking $(3.4$ vs. $3.2 \mathrm{~cm}, \mathrm{P}=0.024)$, a larger amount of pleural fluid drained $(1,093$ vs. $903.5 \mathrm{~mL}, \mathrm{P}=0.01$ ), and were more likely to undergo bilateral procedures ( $7.6 \%$ vs. $2.3 \%, \mathrm{P}=0.044)$. In the multivariate regression analysis, volume of pleural fluid drained was significantly associated with the development of pneumothorax (OR, 1.001, 95\% CI, 1-1.001; P=0.042).

Conclusions: The incidence of pneumothorax following pre-procedural US-guided thoracentesis was relatively high in the present study. The amount of pleural fluid drained was the main factor associated with the risk of developing pneumothorax in these cases.
\end{abstract}

Keywords: Pleural effusion; thoracentesis; pneumothorax

Submitted Sep 20, 2019. Accepted for publication Nov 21, 2019.

doi: $10.21037 /$ jtd.2019.12.39

View this article at: http://dx.doi.org/10.21037/jtd.2019.12.39

\section{Introduction}

Thoracentesis is a commonly performed procedure for diagnostic and therapeutic purposes (1-3). It is considered a low risk and generally safe procedure. Pneumothorax following thoracentesis is associated with increased morbidity, mortality and length of hospital stay $(4,5)$. In approximately $50 \%$ of patients who develop pneumothorax, chest tube insertion is required, resulting in an additional increase in hospital stay length and a substantial economic burden $(4,6,7)$. The incidence of thoracentesis-related pneumothorax has been reported in previous studies. A systematic review consisting of 9,230 thoracentesis reported a $0.61 \%$ incidence of pneumothorax (8). One of the factors 
responsible for the decrease in thoracentesis-related pneumothorax over time is the use of ultrasound (US), which enables the operator to assess the characteristics of a pleural effusion and to identify the most accessible area of pleural fluid (9). Previous studies have reported an incidence of $4-30 \%$ for thoracenteses -related pneumothorax without the use of US and a $1.3-6.7 \%$ with the use of US (10-12). Additional studies have demonstrated that use of US is associated with decreased rate of pneumothorax (13-15). It has not yet been determined whether real-time US guidance offers an additional benefit beyond pre-procedural US regarding thoracentesis-related pneumothorax.

Risk factors for thoracentesis-related pneumothorax have been investigated. It has been found that underweight patients were more likely to experience pneumothorax (16). Multiple needle passes have also been associated with higher rates of pneumothorax (17). It has previously been demonstrated that the risk of pneumothorax rises with drainage of volumes greater than $1,500 \mathrm{~mL}$ compared to drainage of less than $1,500 \mathrm{~mL}$ (16). Operator experience has also been found to be associated with the rate of thoracentesis-related pneumothorax. A previous study found a pneumothorax rate of $3.9 \%$ in procedures performed by experienced operators compared with a rate of $8.5 \%$ in procedures performed by less-experienced operators (17).

The incidence and risk factors of pneumothorax following pre-procedural US-guided thoracentesis have not been investigated. It has not yet been determined whether real-time US guidance offers an additional benefit beyond pre-procedural US regarding thoracentesis-related pneumothorax. Therefore, in the present study, we aimed to determine the incidence and risk factors of thoracentesisrelated pneumothorax using pre-procedural US guidance.

\section{Methods}

We performed a retrospective study of patients who underwent pre-procedural US-guided thoracentesis at Sheba Medical Center, Israel, between January 2016 and December 2018. The electronic medical records of consecutive patients who underwent thoracentesis were examined. Pre-procedural US was routinely used for fluid localization. Chest X-rays were ordered routinely following thoracentesis. The incidence of pneumothorax following thoracentesis was calculated. Diagnosis of pneumothorax was based on chest $\mathrm{X}$-rays done within approximately 2 hours of the procedure.

The baseline clinical and demographic characteristics of all patients were collected. They included age, sex, height, weight, body mass index (BMI), comorbidities, laboratory findings and usage of anticoagulation.

Thoracentesis-associated factors collected included the purpose of the procedure (diagnostic $v s$. therapeutic), depth of pleural marking, rate of dry taps, bilateral procedures and previous thoracentesis, amount of fluid drained and whether the pleural fluid was a transudate or an exudate. Additional information included the etiology of the pleural effusion.

Outcomes evaluated include rate of lung re-expansion, chest tube insertion, intensive care unit (ICU) admission, in-hospital mortality rate and length of hospital stay. Comparison of these parameters between patients with and without pneumothorax was performed. We used multivariate logistic regression analysis to evaluate predictors of development of pneumothorax following thoracentesis.

The research protocol was approved by the Sheba Medical Center review board.

\section{Statistical analysis}

Data were analyzed with Statistical R statistical software (The R Foundation for Statistical Computing) version 3.5.1. Continuous variables were expressed as mean \pm standard deviation. Categorical variables were expressed as frequencies (percentage). The clinical, demographic, thoracentesis-associated characteristics and outcomes of study subjects were compared with Chi-Square tests for categorical variables and independent $t$-tests for continuous variables between patients with and without pneumothorax.

Multivariable Cox proportional hazards regression analysis was used to determine the hazard ratio and significance of baseline factors in developing pneumothorax. All tests were two-tailed, with $\mathrm{P}$ values $<0.05$ being considered as significant.

\section{Results}

During the study period, 550 patients with pleural effusions underwent pre-procedural US-guided thoracentesis. Among these, 66 (12\%) developed pneumothorax following thoracentesis. The mean age of the patients was 66.8 $( \pm 15)$ years, and $327(59.7 \%)$ were males. The incidence of congestive heart failure (CHF) was significantly higher among patients who developed pneumothorax compared to patients who did not develop pneumothorax (47\% vs. $32.2 \%, \mathrm{P}=0.026$ ). Other baseline demographic, clinical and 
Table 1 Baseline clinical and demographic characteristics of patients with and without pneumothorax following thoracentesis

\begin{tabular}{|c|c|c|c|c|}
\hline Variable & $\begin{array}{l}\text { All procedures } \\
\qquad(\mathrm{n}=550)\end{array}$ & $\begin{array}{l}\text { Procedures without pneumothorax } \\
\qquad(\mathrm{n}=484)\end{array}$ & $\begin{array}{l}\text { Procedures with pneumothorax } \\
\qquad(\mathrm{n}=66)\end{array}$ & $P$ value \\
\hline Age, years $( \pm S D)$ & $66.8( \pm 15)$ & $63.4( \pm 15.2)$ & $69.4( \pm 13.2)$ & 0.125 \\
\hline Weight, kg $( \pm S D)$ & $72.3( \pm 20.3)$ & $71.1( \pm 19)$ & $75.8( \pm 27.7)$ & 0.137 \\
\hline Height, cm ( $\pm S D)$ & $160.6( \pm 28.4)$ & $160.8( \pm 28.3)$ & $160.3( \pm 29.6)$ & 0.92 \\
\hline HTN (\%) & 309 (56.2) & 237 (49.0) & $36(54.5)$ & 0.878 \\
\hline DM (\%) & $174(31.6)$ & $154(31.8)$ & $20(30.3)$ & 0.915 \\
\hline Cirrhosis (\%) & $7(1.3)$ & $7(1.4)$ & 0 & 0.691 \\
\hline $\operatorname{INR}( \pm \mathrm{SD})$ & $1.24( \pm 0.33)$ & $1.23( \pm 0.3)$ & $1.3( \pm 0.46)$ & 0.168 \\
\hline aPTT, seconds $( \pm \mathrm{SD})$ & $31.4( \pm 8.1)$ & $31.5( \pm 8.3)$ & $30.6( \pm 6.7)$ & 0.446 \\
\hline Anticoagulation* [\%] & $221[40]$ & $196[40]$ & 25 [38] & 0.9 \\
\hline
\end{tabular}

*, includes low molecular weight heparin, warfarin and new oral anticoagulants. SD, standard deviation; BMI, body mass index; HTN, hypertension; DM, diabetes mellitus; CHF, congestive heart failure; INR, international normalized ratio; aPTT, activated partial thromboplastin time.

laboratory characteristics were similar between patients who developed pneumothorax and those who did not (Table 1).

Among all thoracentesis, 143 (26\%) were performed for a diagnostic purpose and 407 (74\%) were performed for a therapeutic purpose. Bilateral procedures were performed in $16(3 \%)$ patients. The most common etiologies of pleural effusion were malignancy (54.4\%) and CHF (20\%).

Comparison between patients with and without pneumothorax regarding thoracentesis-associated factors is presented in Table 2. Compared to patients who did not develop pneumothorax, those who developed pneumothorax had a smaller depth of pleural fluid marking $(3.2 v s .3 .4 \mathrm{~cm}$, $\mathrm{P}=0.024)$.

The amount of fluid drained was larger among patients who developed pneumothorax compared to those who did not $(1,093$ vs. $903.5 \mathrm{cc}, \mathrm{P}=0.01)$.

Compared to patients who did not develop pneumothorax, those who developed pneumothorax were more like to undergo bilateral procedures rather than a unilateral procedure ( $7.6 \%$ vs. $2.3 \%, \mathrm{P}=0.044)$.

Outcomes of patients who underwent thoracentesis according to the development of pneumothorax are presented in Table 3. Among the patients who developed pneumothorax, 12 (18\%) had re-expansion of the lung.

The rate of chest tube insertion was higher among patient who developed pneumothorax compared to those who did not $(18.2 \%$ vs. $0.8 \%, \mathrm{P}<0.001)$. In-hospital mortality was higher among patients who developed pneumothorax compared to those who did not (18.2\% vs. $8.9 \%, \mathrm{P}=0.032)$. Length of hospital stay was longer among patients who developed pneumothorax compared to those who did not (14.2 vs. 8.3 days, $\mathrm{P}=0.007)$. Age, male sex, BMI, CHF (etiology of pleural effusion), volume of pleural fluid drained and depth of pleural marking were included in the multivariate regression analysis to identify independent factors associated with the development of pneumothorax. Volume of pleural fluid drained was found to be independently associated with the development of pneumothorax following thoracentesis (OR, 1.001, 95\% CI, 1-1.001; $\mathrm{P}=0.042$ ) (Table 4).

\section{Discussion}

The overall rate of pneumothorax following thoracentesis in 
Table 2 Thoracentesis associated factors of study population-comparison between patients with and without pneumothorax

\begin{tabular}{|c|c|c|c|c|}
\hline Variable & $\begin{array}{l}\text { All procedures } \\
\qquad(\mathrm{n}=550)\end{array}$ & $\begin{array}{l}\text { Procedures without pneumothorax } \\
\qquad(\mathrm{n}=484)\end{array}$ & $\begin{array}{l}\text { Procedures with pneumothorax } \\
\qquad(\mathrm{n}=66)\end{array}$ & $P$ value \\
\hline Diagnostic (\%) & $143[26]$ & $130[27]$ & $13(19.7)$ & 0.274 \\
\hline Therapeutic (\%) & $407[74]$ & $354(73.1)$ & $53(80.3)$ & 0.274 \\
\hline Bilateral procedure (\%) & $16[3]$ & $11(2.3)$ & $5(7.6)$ & 0.044 \\
\hline Previous paracentesis (\%) & $332(60.4)$ & $284(58.7)$ & $48(72.7)$ & 0.066 \\
\hline$\geq 3$ previous paracentesis $(\%)$ & $174(31.6)$ & $148(30.6)$ & $26(39.4)$ & 0.192 \\
\hline Invasive/non-invasive ventilation (\%) & $16(2.9)$ & $15(3.1)$ & $1(1.5)$ & 0.743 \\
\hline \multicolumn{5}{|l|}{ Etiology of pleural effusion } \\
\hline $\mathrm{CHF}(\%)$ & $109[20]$ & $90(18.6)$ & $19(28.8)$ & 0.074 \\
\hline Infectious (\%) & $28(5.1)$ & $25(5.2)$ & $3(4.5)$ & 1 \\
\hline Malignant (\%) & $299(54.4)$ & $260(53.7)$ & $39(59.1)$ & 0.49 \\
\hline Post-operative (\%) & $14(2.5)$ & $13(2.7)$ & $1(1.5)$ & 0.881 \\
\hline Other (\%) & $15(2.7)$ & $14(2.9)$ & $1(1.5)$ & 0.809 \\
\hline Unknown (\%) & 77 [14] & $72(14.9)$ & $5(7.6)$ & 0.157 \\
\hline
\end{tabular}

$\mathrm{SD}$, standard deviation; CHF, congestive heart failure.

Table 3 Outcome of patients who underwent thoracentesis according to the development of pneumothorax

\begin{tabular}{|c|c|c|c|c|}
\hline Variable & $\begin{array}{l}\text { All procedures } \\
\qquad(\mathrm{n}=550)\end{array}$ & $\begin{array}{l}\text { Procedures without pneumothorax } \\
\qquad(\mathrm{n}=484)\end{array}$ & $\begin{array}{l}\text { Procedures with pneumothorax } \\
\qquad(\mathrm{n}=66)\end{array}$ & $P$ value \\
\hline Chest tube insertion (\%) & $16(2.9)$ & $4(0.8)$ & $12(18.2)$ & $<0.001$ \\
\hline Operation (\%) & $1(0.2)$ & 0 & 0 & 1 \\
\hline Lung re-expansion (\%) & $496[90]$ & $484[100]$ & $12[18]$ & $<0.001$ \\
\hline
\end{tabular}

ICU, intensive care unit.

the present study was $12 \%$. Previous studies demonstrated that thoracentesis-associated pneumothorax occurs in $0.6-30 \%$ of the patients. Ault et al. found a rate of $0.61 \%$ for thoracentesisassociated pneumothorax (8). In their study, thoracentesis was performed with the aid of a portable US for fluid localization through site marking. US was also used to monitor the progress of fluid removed by intermittently interrogating the pleural space throughout the procedure. Seneff et al. found a pneumothorax rate of $11.5 \%$. Only a minority of the procedures were performed under US guidance (11). Gervais et al. found that the rate of pneumothorax following USguided thoracentesis was $2.3 \%$ (12). 
Table 4 Predictors for development of pneumothorax following thoracentesis-multivariate logistic regression analysis

\begin{tabular}{lcc}
\hline Variable & OR $(95 \% \mathrm{Cl})$ & $\mathrm{P}$ value \\
\hline Age & $1.005(0.984-1.027)$ & 0.621 \\
Male sex & $1.493(0.873-2.114)$ & 0.206 \\
BMI & $0.932(0.859-1.005)$ & 0.058 \\
CHF (etiology of pleural effusion) & $1.825(1.16-2.491)$ & 0.077 \\
Volume of fluid drained & $1.001(1-1.001)$ & 0.042 \\
Depth of pleural marking & $0.608(0.079-1.138)$ & 0.066 \\
\hline
\end{tabular}

OR, odd ratio; BMI, body mass index; CHF, congestive heart failure.

Several studies have demonstrated that US-guided thoracentesis is associated with a decrease in the rate of pneumothorax. Grogan et al. demonstrated the direct USguided thoracentesis was associated with fewer pneumothoraxes then procedures that were not US-guided (10).

Barnes et al. assessed whether thoracenteses performed with US-guidance are associated with a lower rate of pneumothorax than those performed without US-guidance. They found that the rate of pneumothorax was $4.9 \%$ in procedures performed with US-guidance compared with a rate of $10.3 \%$ in procedures performed without (14). According to the studies presented herewith, it seems that the rate of thoracentesis-associated pneumothorax in our study is similar to rates of pneumothoraxes associated with thoracentesis performed without US guidance. Further prospective studies should be conducted in order to assess whether real-time US-guidance thoracentesis is superior to pre-procedural US in regards to the risk of developing pneumothorax.

In the present study, we found the rate of CHF was significantly higher among patients who developed pneumothorax than those who did not. However, in the multivariate analysis, CHF by itself did not predict development of pneumothorax. Previous studies have not investigated baseline comorbidities in regards to the risk of developing thoracentesis-associated pneumothorax.

In our study, BMI was lower among patients who developed pneumothorax than those that did not. However, the difference was not statistically significant. Previous studies have demonstrated that underweight is associated with the risk of developing thoracentesis-associated pneumothorax $(8,18)$. The lack of statistically significant difference in BMI between patients who developed pneumothorax and those that did not in our study may be attributed to the small study population in comparison with studies mentioned above. These findings indicate that lean patients are at higher risk for developing pneumothorax, apparently as a result of the shorter distance between the chest wall and the lung.

We assessed the association between thoracentesisrelated factors and development of pneumothorax. The depth of pleural marking has not been evaluated in previous studies. Since US guidance was pre-procedural in our study, as opposed to previous studies, we thought this parameter should be evaluated. We found that patients who developed pneumothorax had a smaller depth of pleural fluid marking than patients who did not develop pneumothorax. We did not find a specific cut-off which was associated with the risk of developing pneumothorax. This finding may suggest that when contemplating the risk-benefit ratio of thoracentesis, pleural marking depth should be taken into consideration. When pleural marking depth is relatively small, executing thoracentesis should be carefully considered, particularly when the procedure is not indispensable.

We also found that the amount of pleural fluid drained was larger among patients who developed pneumothorax than those that did not. This finding is in line with previous studies which has shown that risk of pneumothorax rises with drainage volumes greater than $1,500 \mathrm{~mL}(8)$. It seems reasonable that a small amount of pleural fluid may be associated with a higher risk of developing pneumothorax because of the proximity between the needle tip and the lung. However, according to the findings in our study and a previous study (8), it seems that larger fluid volumes removed are associated with the development of pneumothorax. A possible explanation for this finding is that when a large volume of pleural fluid is removed, a relatively significant decrease in pleural pressure occurs, which may result in rupture of the visceral pleura and development of pneumothorax. 
In our study bilateral procedures were associated with the development of pneumothorax. Previous studies regarding this issue are inconsistent.

The study by Cho et al. showed bilateral procedures were not associated with the risk of pneumothorax. It should be noted that only 3 patients in their study underwent a bilateral procedure (18). Conversely, in the study by Ault et al., bilateral procedures were associated with a lower risk of pneumothorax (8). The explanation for this finding proposed by the authors was that patient factors that reduce the risk of pneumothorax in the first side are then conferred to the procedure on the other side. In some cases, bilateral procedures are necessary for the purpose of improving pulmonary function and weaning patients off mechanical ventilation. Based on the finding in our study, in cases where a bilateral procedure is not necessary, it seems reasonable to perform a unilateral procedure. The execution of a contralateral procedure may be delayed until ensuring no procedural complications have occurred in the first procedure.

In the multivariate analysis, we found that the volume of pleural fluid drained was significantly associated with the development of pneumothorax. This finding is line with the British Thoracic Society (BTS) guidelines, which suggested that thoracentesis should be stopped when $1,500 \mathrm{ml}$ has been aspirated, in order to reduce the risk of pneumothorax (19). According to the offered mechanism mentioned above, limiting the amount of fluid removed may decrease the rate of pneumothorax, rather than affecting the size of pneumothorax.

In our study, development of pneumothorax following thoracentesis resulted in an increased mortality and an increase in hospital stay length. These findings are in accordance with findings in previous studies (4-7), and demonstrate that thoracentesis-associated pneumothorax is associated with increased morbidity, mortality and economic burden.

Our study has several limitations. First, it is a retrospective study. In addition, data regarding operator experience was not available. Therefore, we could not assess the association between operator experience and rate of pneumothorax.

To the best of our knowledge, this is the first study that evaluated the incidence and risk factors of thoracentesisrelated pneumothorax using pre-procedural US-guidance. Further studies are required to determine whether realtime US guidance thoracentesis is superior to USguidance thoracentesis regarding the risk of developing pneumothorax.

In conclusion, we have demonstrated that the incidence of pneumothorax following thoracentesis using preprocedural US-guidance is relatively high. In addition, we have shown that the amount of pleural fluid drained is the main factor associated with the risk of developing pneumothorax in these cases.

\section{Acknowledgments}

Funding: None.

\section{Footnote}

Conflicts of Interest: The authors have no conflicts of interest to declare.

Ethical Statement: The authors are accountable for all aspects of the work in ensuring that questions related to the accuracy or integrity of any part of the work are appropriately investigated and resolved. The Local Ethics Committee approved the study (Referral number: 4988-10). Written informed consent was obtained from the patient for publication of this manuscript and any accompanying images.

Open Access Statement: This is an Open Access article distributed in accordance with the Creative Commons Attribution-NonCommercial-NoDerivs 4.0 International License (CC BY-NC-ND 4.0), which permits the noncommercial replication and distribution of the article with the strict proviso that no changes or edits are made and the original work is properly cited (including links to both the formal publication through the relevant DOI and the license). See: https://creativecommons.org/licenses/by-nc-nd/4.0/.

\section{References}

1. Owings MF, Kozak LJ. Ambulatory and inpatient procedures in the United States, 1996. Vital Health Stat 13 1998;(139):1-119.

2. Light RW. Pleural effusions. Med Clin North Am 2011;95:1055-70.

3. Feller-Kopman D. Ultrasound-guided thoracentesis. Chest 2006;129:1709-14.

4. Gordon CE, Feller-Kopman D, Balk EM, et al. Pneumothorax following thoracentesis: a systematic review and meta-analysis. Arch Intern Med 2010;170:332-9. 
5. Mercaldi CJ, Lanes SF. Ultrasound guidance decreases complications and improves the cost of care among patients undergoing thoracentesis and paracentesis. Chest 2013;143:532-8.

6. Sassoon CS, Light RW, O'Hara VS, et al. Iatrogenic pneumothorax: etiology and morbidity. Results of a Department of Veterans Affairs Cooperative Study. Respiration 1992;59:215-20.

7. Despars JA, Sassoon CS, Light RW. Significance of iatrogenic pneumothoraces. Chest 1994;105:1147-50.

8. Ault MJ, Rosen BT, Scher J, et al. Thoracentesis outcomes: a 12-year experience. Thorax 2015;70:127-32.

9. Yang PC, Luh KT, Chang DB, et al. Value of sonography in determining the nature of pleural effusion: analysis of 320 cases. AJR Am J Roentgenol 1992;159:29-33.

10. Grogan DR, Irwin RS, Channick R, et al. Complications associated with thoracentesis. A prospective, randomized study comparing three different methods. Arch Intern Med 1990;150:873-7.

11. Seneff MG, Corwin RW, Gold LH, et al. Complications associated with thoracocentesis. Chest 1986;90:97-100.

12. Gervais DA, Petersein A, Lee MJ, et al. US-guided thoracentesis: requirement for postprocedure chest

Cite this article as: Shechtman L, Shrem M, Kleinbaum Y, Bornstein G, Gilad L, Grossman C. Incidence and risk factors of pneumothorax following pre-procedural ultrasound-guided thoracentesis. J Thorac Dis 2020;12(3):942-948. doi: 10.21037/ jtd.2019.12.39 radiography in patients who receive mechanical ventilation versus patients who breathe spontaneously. Radiology 1997;204:503-6.

13. Jones PW, Moyers JP, Rogers JT, et al. Ultrasound-guided thoracentesis: is it a safer method? Chest 2003;123:418-23.

14. Barnes TW, Morgenthaler TI, Olson EJ, et al. Sonographically guided thoracentesis and rate of pneumothorax. J Clin Ultrasound 2005;33:442-6.

15. Raptopoulos V, Davis LM, Lee G, et al. Factors affecting the development of pneumothorax associated with thoracentesis. AJR Am J Roentgenol 1991;156:917-20.

16. Daniels CE, Ryu JH. Improving the safety of thoracentesis. Curr Opin Pulm Med 2011;17:232-6.

17. Josephson T, Nordenskjold CA, Larsson J, et al. Amount drained at ultrasound-guided thoracentesis and risk of pneumothorax. Acta Radiol 2009;50:42-7.

18. Cho HY, Ko BS, Choi HJ, et al. Incidence and risk factors of iatrogenic pneumothorax after thoracentesis in emergency department settings. J Thorac Dis 2017;9:3728-34.

19. Havelock T, Teoh R, Laws D, et al. Pleural procedures and thoracic ultrasound: British Thoracic Society Pleural Disease Guideline 2010. Thorax 2010;65 Suppl 2:ii61-76. 